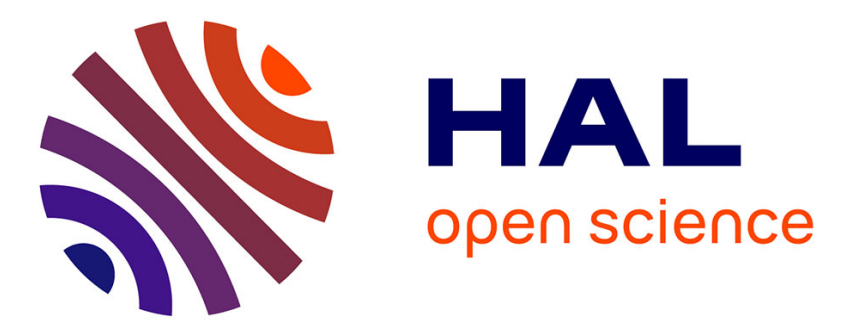

\title{
Former les enseignants pour accroître leur satisfaction au travail: propositions théoriques et illustrations empiriques
}

\author{
Sébastien Chalies, Jérôme Amathieu, Stefano Bertone
}

\section{- To cite this version:}

Sébastien Chalies, Jérôme Amathieu, Stefano Bertone. Former les enseignants pour accroître leur satisfaction au travail: propositions théoriques et illustrations empiriques. Le travail humain, 2013, 76 (4), 10.3917/th.764.0309 . hal-01452577

\section{HAL Id: hal-01452577 \\ https://hal.science/hal-01452577}

Submitted on 6 Feb 2017

HAL is a multi-disciplinary open access archive for the deposit and dissemination of scientific research documents, whether they are published or not. The documents may come from teaching and research institutions in France or abroad, or from public or private research centers.
L'archive ouverte pluridisciplinaire HAL, est destinée au dépôt et à la diffusion de documents scientifiques de niveau recherche, publiés ou non, émanant des établissements d'enseignement et de recherche français ou étrangers, des laboratoires publics ou privés. 


\title{
THÉORIES ET MÉTHODOLOGIES THEORIES AND METHODOLOGIES
}

\author{
FORMER LES ENSEIGNANTS POUR ACCROÎTRE \\ LEUR SATISFACTION AU TRAVAIL : PROPOSITIONS \\ THÉORIQUES ET ILLUSTRATIONS EMPIRIQUES
}

\author{
par Sébastien Chaliès ${ }^{1}$, Jérome Amathieu ${ }^{2}$, Stéfano Bertone ${ }^{3}$
}

SUMMARY

TRAINING TEACHERS TO IMPROVE THEIR JOB SATISFACTION : THEORETICAL PROPOSALS AND EMPIRICAL ILLUSTRATIONS

Fob satisfaction has become an issue of some importance, particularly as it is now widely acknowledged that it has an impact on health, particularly for teachers. Training is one of the main ways to increase job satisfaction but, paradoxically, few works have focused on the causal link between teachers'level of job satisfaction and the help that can be offered to them by training programs. Moreover, these studies have been quantitative experiments working from a positivist and causalist perspective. This study seeks to determine the circumstances in which teacher training has an impact on the job satisfaction of the trainees. It is structured in three parts. The first part presents a conceptualization of training based on postulates from a general theory of learning (Berducci, 2004; Nelson, 2008) and collective action (Wittgenstein, 2004). This conceptualization provides the basis for an original definition of job satisfaction at the scale of training and/or work situations and the real activities of the actors who are involved. With reference to a hypothesis developed in the Clinic of Activity, according to which "work well done is the source for health at work" (Clot, 2010, p. 21), job satisfaction is considered to be correlated with the adequacy of the work from the perspective of those who are doing it. In other terms, job satisfaction can be correlated with the employee's "retrospective authentification" (Chauviré, 2010) of what was expected. The second part presents a case study to illustrate and then discuss the two main training circumstances in which the instructors' training activity had an influence on the job satisfaction of preservice teachers. Last, the third section discusses the three conditions that must be met in order to arrange teacher training order to improve job satisfaction: re-thinking the principle of alternating work/study, working toward the emergence of criteria for satisfaction by teaching work rules, and accentuating the quality of work rather than its yield.

Key words: Training, alternating work/study, job satisfaction, work, work rules, preservice teachers.

1. UMR EFTS, Université de Toulouse, IUFM, 118 Route de Narbonne 31078 Toulouse - sebastien.chalies@univ-tlse2.fr

2. UMR EFTS, Université de Toulouse, IUFM, 118 Route de Narbonne 31078 Toulouse-jamathieu@hotmail.fr

3. EA DIMPS, Université de la Réunion, IUFM, 117 Av G. Ailleret 97430 Le Tampon - bertone.stefano@gmail.com 


\section{INTRODUCTION}

Encore aujourd'hui, la satisfaction professionnelle est généralement considérée comme la conséquence du fait que les travailleurs aiment ou non leur travail et, en le réalisant, y développent un sentiment de bien être (Weiss, 2002). Ce sentiment est directement influencé par la possibilité offerte aux travailleurs d'accomplir certaines de leurs aspirations au travail (Fabra \& Camison, 2009), notamment celles de pouvoir «bien le faire " (Clot, 2010) et de s’y développer (Bertrand, Peters, Pérée, \& Hansez, 2010). L'intérêt porté à la satisfaction professionnelle dans la littérature est d'autant plus important qu'il est actuellement admis qu'elle a une influence sur la santé des travailleurs (Faragher, Cass, \& Cooper, 2005) et des enseignants en particulier (Ma \& MacMillan, 1999). En cas d'insatisfaction professionnelle marquée et récurrente, ces derniers présentent des troubles plus ou moins sévères de détresse psychologique, tels que l'insomnie, l'anxiété, le stress voire la dépression (Demirtas, 2010). Plus inquiétant, ces troubles peuvent dans certaines circonstances engendrer une situation de "burnout" (Hastings \& Bham, 2003), c'est à dire une situation d'épuisement psychologique et physique qui les conduit à arrêter momentanément leur travail voire à l'abandonner (Hansez, Bertrand, De Keyser, \& Pérée, 2005). Inversement, des travaux montrent que l'accroissement de la satisfaction professionnelle des enseignants améliore leur santé notamment psychologique (Moè, Pazzaglia, \& Ronconi, 2010). Lorsqu'ils sont satisfaits de leur travail, ils développent alors un sentiment d'efficacité plus marqué et, en retour, réduisent leur stress (Kyriacou, 2001). Ils s'engagent aussi de façon plus prononcée et enthousiaste dans leur travail auprès des élèves (Ciftci, Ozkan Ozgun, \& Erden, 2011).

Sur la base de ces constats, des travaux ont été menés pour identifier les facteurs sur lesquels jouer pour accroittre la satisfaction professionnelle et par-là même la santé des enseignants (Roussel, 2001). Parmi les modèles les plus exploités, celui de Duffy \& Lent (2009) propose de considérer la satisfaction professionnelle sous l'influence de cinq facteurs interdépendants : le type de personnalité du travailleur (en termes d'estime de soi, de motivation, d'âge, de sexe voire de satisfaction personnelle), la qualité du but poursuivi (un but élevé étant plutôt source de satisfaction professionnelle), les aides mises à sa disposition pour atteindre le but poursuivi, les conditions humaines et matérielles de travail, et le sentiment d'efficacité personnelle. Parmi ces facteurs, les deux derniers ont une influence particulièrement significative sur la satisfaction professionnelle des enseignants.

La satisfaction professionnelle des enseignants est en effet tout d'abord particulièrement influencée par leurs conditions humaines et matérielles d'enseignement, c'est-à-dire par les conditions de travail offertes par leurs établissements scolaires (Huang \& Waxman, 2009). Parmi ces conditions, on peut relever : (i) la possibilité qui leur est offerte de participer à la gouvernance de l'établissement et par-là même de développer un véritable sentiment d'appartenance à ce dernier (Smylie, 1994), (ii) la reconnaissance de leur travail par leur hiérarchie (Papanastasiou \& Zembylas, 2005), leurs collègues (Shen, Leslie, Spybrook, \& Ma, 2011), les parents (Day, 
Sammons, Stobard, Kington, \& Gu, 2007) et/ou leurs élèves (Skaalvik \& Skaalvik, 2011), (iii) le caractère adapté de leur charge de travail (Liu \& Ramsey, 2008), (iv) le degré d'autonomie qui leur accordé dans la réalisation de leur enseignement (Pearson \& Moomaw, 2006), et (v) les possibilités d'interagir avec d'autres enseignants, voire d'engager des projets collectifs (Brunetti, 2001).

La satisfaction professionnelle est aussi influencée par le sentiment d'efficacité personnelle des enseignants lors de la réalisation de leur travail (Caprara, Barbaranelli, Steca, \& Malone, 2006). Il est en effet possible d'établir un lien de causalité entre l'efficacité personnelle perçue au travail et la satisfaction professionnelle. Ainsi, plus les enseignants se sentent efficaces au travail plus ils éprouvent une satisfaction (Moe et al., 2010).

Selon Duffy \& Lent (2009), les aides mises à la disposition des enseignants pour qu'ils puissent atteindre les buts poursuivis dans leur travail sont aussi un facteur pouvant influencer leur satisfaction professionnelle. Paradoxalement, ces aides ont fait l'objet de peu de recherches. Lorsqu'elles sont évoquées, c'est principalement en termes de pistes pour aménager autrement le travail. Menées exclusivement sur la base de dispositifs expérimentaux quantitatifs, quelques études ont toutefois établi un lien de causalité entre la satisfaction des enseignants au travail et la possibilité qui leur est offerte d'être aidés, notamment par la mise en place de dispositifs de formation (Rots, Aelterman, Devos, \& Vlerick, 2010). Ces études montrent principalement que la satisfaction professionnelle des enseignants croît d'autant plus que des possibilités de soutien, d'entraide et de coopération leur sont proposées au sein de leurs établissements scolaires.

A notre connaissance, aucune des études présentées précédemment, pour une large majorité réalisées en vue d'identifier d'éventuelles relations causales conformément au paradigme positiviste (Miossec \& Clot, 2011), renseigne la manière dont les aides mises à disposition des enseignants influencent leur satisfaction professionnelle. L'objet de la présente étude est de chercher plus précisément à identifier les circonstances dans lesquelles une activité de formation menée par des formateurs a une influence sur la satisfaction professionnelle de formés. Pour cela, elle va s'attacher successivement à (i) présenter une conceptualisation de la formation au cœur de laquelle une définition originale de la satisfaction professionnelle est envisageable à l'échelle des situations de formation et/ou de travail et des activités réelles des acteurs y étant impliqués, et (ii) à illustrer puis discuter à partir des résultats d'une étude de cas, les deux principales circonstances de formation dans lesquelles une activité de formation a eu une influence sur la satisfaction professionnelle d'enseignants novices (EN).

\section{CADRE THEORIQUE}

Dans cette étude, le principe de l'alternance entre situation de formation au travail et situation de travail a été conceptualisé à partir de postulats empruntés à une théorie générale de l'apprentissage (Berducci, 
2004 ; Nelson, 2008) et de l'action collective (Wittgenstein, 2004). Trois hypothèses empruntées à cette conceptualisation (pour plus de détails : Bertone, Chaliès, \& Clot, 2009) ont été retenues.

\section{II.1. Premiere hypothese : TRAVAiller ET SE FORMER NECESSITENT DE S'ENGAGER DANS UN DOUBLE REGIME DE REFLEXIVITÉ}

Dans la lecture théorique proposée, travailler revient à mener des actions gouvernées par des règles et/ou à réaliser des actions de suivi des règles. Ces règles ne sont rien d'autre que des expériences situées normatives acceptées par la communauté professionnelle (Wittgenstein, 2004). Autrement dit, elles n'ont d'autorité que si le travailleur décide de les suivre ou s'il est encouragé, par exemple par des formateurs, à les suivre (Descombes, 2004). Lorsqu'il travaille, chaque acteur s'engage plus précisément dans un double registre de réflexivité par rapport aux règles (Ogien, 2007). Dans certaines situations professionnelles, il est engagé dans un régime de réflexivité de l'ordre de l'inhérence. Il mène alors des "actions gouvernées " par les règles (Ogien, 2007). Il agit donc selon ces dernières qui, à l'instant considéré lui sont soit préconscientes et potentiellement dicibles parce qu'apprises lors d'une formation réflexive antérieure, soit non conscientes et par-là même non dicibles. Dans un grand nombre de situations de travail, le travailleur réalise en effet des actions "gouvernées" par les règles dont l'apprentissage s'est fait implicitement (Lave \& Wenger, 1991) par l'intermédiaire d'interactions non verbales et/ou d'alignements informels avec la pratique des autres membres (Rogoff, Matusov, \& White, 1996) au cours de sa participation aux pratiques professionnelles constitutives de la communauté. Dans d'autres situations professionnelles, le travailleur est engagé dans un régime de réflexivité de l'ordre de l'appréhension de l'inhérence : il réalise alors des actions de "suivi " des règles (Ogien, 2007). Il agit donc en conformité avec ces dernières qui, à l'instant considéré, sont conscientes et dicibles parce qu'apprises lors d'une formation réflexive antérieure au cours de laquelle il a appris avec l'aide d'un ou plusieurs formateurs à les rendre intelligibles par l'intermédiaire de réélaborations discursives. Le principe de formation par alternance entre formation au travail et travail étant principalement fondé à partir de ce second régime de réflexivité, nous le détaillons ci-dessous.

\section{II.2. Deuxieme hypothese : TOUTE FORMATION PROFESSIONNELlE REFLEXIVE REPOSE SUR L'APPRENTISSAGE PUIS L'INTERPRETATION DE RĖGLES PAR LES FORMÉS}

La possibilité d'un engagement des formés dans une pratique réflexive à propos de leur travail nécessite un apprentissage préalable de règles leur permettant de signifier correctement les expériences professionnelles qu'ils ont pu vivre, c'est-à-dire comme il est attendu de le faire au sein de la communauté professionnelle considérée. Ce qui permet ici de caractériser le couplage d'activités "formation / apprentissage» ou «enseignement / apprentissage " est l'idée d'une tutelle intentionnelle du formé, le formateur 
étendant (prêtant) ses intentions à celui-ci, qui n'en dispose pas (encore). En d'autres termes, le formateur qui " prête des intentions " à un apprenant, lui fournit l'arrière plan cognitif dont il ne dispose pas encore pour être véritablement un acteur intentionnel. Le formé se limite, au début, à réagir à des stimuli ou à imiter des actions. C'est précisément de ces actions que vont émerger les préoccupations et les intentions du futur " acteur intentionnel ", qui deviendra ainsi un tuteur potentiel à son tour (Berducci, 2004). On trouve ce point de vue clairement exprimé par Berducci (2004):

"Ces novices doivent premièrement s'engager dans un apprentissage ostensiflbéhavioriste (ostensivelbehavioristic training) au cours duquel ils miment l'obeiissance à des règles et agissent, mais sans intention (they mimic rule-obeying, and act, but without intention). La possibilité d'une action sans intention existe (Rubinstein, 1981, p. 111), et c'est ce type d'action qui constitue le fait de se conformer aux règles. C'est l'apprentissage qui change les actions non intentionnelles en intentionnelles et donc les actions obéissant réellement à des règles. Un novice intègre le fait que la compréhension des règles requiert la familiarisation à des pratiques sociales par l'apprentissage (Williams, 1999, p. 203)." (Berducci, 2004, p.340, traduit de l'anglais par les auteurs).

C'est sur la base de cette conception qu'il sera considéré par la suite que tout apprentissage est clôturé lorsqu'une action obéissant aux règles a été réalisée par un formé sous tutelle intentionnelle. En outre, dans la mesure où, dans cette approche, il est considéré que "l'intention c'est l'action " (Descombes, 1996; Gauvry, 2010), il est possible d'avancer que l'apprentissage d'une action obéissant aux règles ouvre l'accès au formé (de façon consubstantielle) non seulement à l'action conforme, mais également à l'intention qui lui est habituellement associée par le tuteur et qui lui donne satisfaction.

Cet apprentissage nécessite l'engagement des formateurs dans une activité "d'enseignement ostensif" (Wittgenstein, 2004) par laquelle ils fondent la signification des expériences professionnelles considérées comme exemplaires. Pour ce faire, ils dressent pour chacune de ces expériences enseignées un "lien de signification" (Bertone et al., 2009) entre, (i) l'expérience langagière visant à la nommer, (ii) les exemples décrits et/ou montrés en correspondance et (iii) les résultats qui y sont usuellement associés dans la communauté. Ces liens de significations enseignés, et donc initialement non construits par une pratique réflexive autonome, servent ensuite de véritables expériences ou "mètre étalon" (Williams, 2002) lorsque les formés agissent dans de nouvelles situations de travail ou de formation au travail. Ces derniers s'appuient alors en effet sur ces échantillons d'expériences exemplaires ostensiblement montrés pour signifier et juger les événements rencontrés, voire essayer de s'y adapter. L'idée d'un échantillonnage de l'expérience de travail pendant la formation peut être rattachée à celle de l'existence d'une sorte de "grammaire sociale » du métier (Laugier \& Chauviré, 2006), que possèdent et mobilisent les membres d'une communauté professionnelle donnée. Cette grammaire de l'expérience de travail peut faire l'objet d'un enseignement par les formateurs et d'une familiarisation pour les formés qui passe par des activités multiples telles que entre autres la description des faits observables ou à observer, l'identification "d'aspects " (Chauviré, 2010) saillants 
à considérer ou encore l'introduction de préférences perceptives rendant "parlantes » et non équivalentes les circonstances vécues dans l'exercice du métier (Narboux, 2003).

Les formateurs ne peuvent toutefois se suffire de cette activité d'enseignement. Ils doivent ensuite s'engager dans une activité d'accompagnement des premiers suivis par les formés des règles préalablement enseignées dans de nouvelles situations de formation et/ou de travail en classe. Par cet accompagnement, ils visent à rendre possible mais aussi et surtout à faire en sorte que ces premiers suivis aboutissent aux résultats attendus y étant habituellement associés. Ce n'est qu'au travers du constat de ces résultats attendus que les formés peuvent associer consubstantiellement une intention professionnelle aux règles enseignées (Cash, 2009). Autrement dit, suivant correctement les règles préalablement enseignées et constatant les résultats qui leur sont usuellement associés dans la communauté professionnelle, les formés finalisent leur apprentissage et peuvent alors se développer à partir de celles-ci (Nelson, 2008). Théoriquement, lors de ces premiers suivis, les formateurs "contrôlent" (Nelson, 2008) la conformité des premiers suivis des règles enseignées par les formés et s'engagent si nécessaire dans une activité d'"explications ostensives " (Davis, 2009). Ils s'efforcent alors de multiplier les exemples décrits et/ ou montrés pouvant être associés aux règles enseignées de sorte de lever les éventuelles incompréhensions ou mésinterprétations des formés et leur permettre, au final, de les suivre seuls et de façon acceptable, c'est-à-dire comme convenu dans la communauté professionnelle.

Lorsque les règles sont apprises, leurs suivis par les formés dans de nouvelles situations de travail et/ou de formation nécessitent leur interprétation (Winch, 2009). Ces suivis renvoient en effet à un usage extensif des liens de signification construits et appris sur la base de l'identification par les formés d'un "air de famille " (Wittgenstein, 2004) entre les circonstances constitutives de la situation en cours et celles constitutives des situations dans lesquelles les règles ont été apprises. À partir des liens de signification appris, les formés ordonnent alors finalement un réseau complexe de ressemblances, comprennent la nouvelle situation en cours, et in fine parviennent à produire des activités conformes aux attentes de la communauté, tout en s'émancipant progressivement de son contrôle. Ces situations de développement ne sont donc plus l'occasion pour les formateurs de contrôler le caractère adéquat ou inadéquat des significations associées par les formés aux événements rencontrés. Elles leur permettent plutôt d'aider ces derniers à s'engager dans des suivis interprétés des règles apprises, c'est-àdire de les aider à en négocier la signification pour mieux en user.

\section{II.3. TROISIÈME HYPOTHÈSE : LA FORMATION ACCROÎT LA SATISFACTION EN PERMETTANT AUX FORMES D'OBTENIR PAR LEUR ACTIVITÉ DES RÉSULTATS ATTENDUS ET UN TRAVAIL RÉALISE SELON LES « RĖGLES DE L'ART »}

Dans la lecture théorique adoptée, le postulat développé en clinique de l'activité selon lequel "le travail bien fait " est "le ressort de la santé au travail» (Clot, 2010, p. 21) a été retenu comme un point d'ancrage 
compatible et complémentaire. en effet, la satisfaction professionnelle a été considérée comme directement corrélée à la justesse du travail du point de vue de ceux qui le réalisent, c'est-à-dire corrélée à une "authentification rétrospective" (Chauviré, 2010) par les travailleurs de ce qui était attendu. Autrement dit, aboutissant aux résultats attendus lors des suivis des règles considérées, les formés s'en trouvent finalement satisfaits. Ce constat peut relever de deux types de circonstances de formation. Tout d'abord, la satisfaction des formés peut être alimentée par les situations de formation concourant à l'apprentissage de nouvelles règles. La satisfaction est alors consubstantielle au constat par les formés des résultats attendus suite à leurs premiers suivis de toute règle préalablement enseignée mais non encore apprise. D'autre part, la satisfaction des formés peut être alimentée par les situations de formation concourant à leurs nouveaux usages des règles préalablement apprises. En élargissant les circonstances dans lesquelles il est possible de "voir " ou "faire jouer " les règles apprises, les formés les ajustent au cours de leurs suivis jusqu'à aboutir à ce qu'ils attendent en termes de résultats. C'est alors qu'ils se trouvent consubstantiellement satisfaits de leur activité.

Cette conception de la satisfaction au travail permet de comprendre (i) l'émergence de nouveaux critères de satisfaction en situation de formation, consubstantiels des actions obéissant à des règles apprises par le formé et (ii) le fait que pour être satisfait du travail, ce dernier doit avoir réussi à obéir aux règles et avoir identifié dans l'action en classe les intentions qui leur sont usuellement associées (et qu'il parvient désormais à poursuivre). De ce fait, comme pour ce qui relève de la tutelle intentionnelle lors des premiers apprentissages, le formé ne peut être satisfait de son expérience de classe à partir de ses seules expériences de classe sans être ipso facto en décalage avec une conception du travail fait "dans les règles de l'art ". Pour être satisfait du travail réalisé, il doit pouvoir déployer sur l'expérience de classe les nouveaux critères de satisfaction appris sous tutelle intentionnelle. A la tutelle intentionnelle lors de la réalisation des actions obéissant aux règles de l'art correspond une tutelle du jugement porté sur la qualité du travail réalisé qui rend la satisfaction du novice subordonnée au jugement de son formateur.

La complémentarité de ce point de vue avec l'approche de la santé au travail développée par Clot (2010) ou Le Blanc (2004), nous semble patente. L'idée partagée, au-delà des nombreuses différences paradigmatiques, est que tout sujet, agent ou opérateur, novice ou expert, se porte bien à la fois lorsqu'il parvient à faire le travail dans "les règles de l'art" ou dans le "genre " du métier qu'il exerce et lorsqu'il parvient, à la suite, à s'écarter de ces règles génériques en les retravaillant et en les mettant à sa main pour répondre aux inattendus du réel de son activité située. Ce double mouvement d'attachement et d'écart aux règles (Le Blanc, 2004), de conformité générique et de différence stylistique (Miossec \& Clot, 2011), constitue le ressort principal de la santé de chacun au travail et de la vitalité du métier. Par ricochet, il est également la source d'une satisfaction subjective quant à la qualité du travail réalisé et à la reconnaissance de l'utilité sociale du produit de son activité (Clot, 2010). Plus complexe que la simple reconnaissance sociale qu'autrui peut apporter à l'activité 
du sujet, cette reconnaissance-là ne se limite pas à un jugement extérieur quant à la conformité du travail à des critères esthétiques ou de rendement (on peut beaucoup produire tout en faisant du travail de mauvaise qualité). Elle est tout autant le fruit de l'accomplissement de soi dans et par l'objet du travail ou le produit de l'activité elle-même. Certes dans un conflit de critères il y a toujours des critères, de même que ces critères doivent nécessairement être à l'origine publics avant d'être considérés par le sujet comme concurrentiels avec d'autres. Mais ils sont consubstantiels à la réalisation même du travail et constituent, dans les deux approches évoquées, le cœur de la satisfaction du sujet et de son accomplissement professionnel. On retrouve clairement dans l'approche clinique de l'activité, des contextes dialogiques d'instruction au sosie (Clot \& Soubiran, 1998) ou d'autoconfrontation croisée (Yvon \& Clot, 2003) jouant un rôle central dans la réélaboration et le développement de l'expérience professionnelle des enseignants. C'est particulièrement sur la base d'une évocation de l'activité réelle lors du travail et de l'engagement des acteurs dans des controverses de métier à propos des façons de faire en classe, que les experts trouvent du répondant et développent leur pouvoir d'agir (Clot, 2008). Ces circonstances dialogiques du développement professionnel et de la reconnaissance par les pairs de l'expérience d'enseignement, autant dans ses composantes génériques que stylistiques, ont été considérées comme pertinentes pour penser la relation que les formés construisent entre les règles du métier enseignant et les jugements de satisfaction/insatisfaction qu'ils énoncent à propos de leur travail. Elles ont également été incarnées dans les entretiens d'autoconfrontation menés dans le cadre de cette étude.

\section{MÉTHODE}

Pour illustrer empiriquement les circonstances de formation dans lesquelles l'activité de formation a eu une influence sur la satisfaction professionnelle des Enseignants Novices (EN), nous nous appuyons sur les résultats d'une étude de cas dont les principales caractéristiques sont présentées ci-après.

\section{III.1. DisPositif SUPPORT A L’ÉTUdE}

Le dispositif de formation support à l'étude de cas était celui dit de l'"Aide aux stagiaires en grande difficulté " proposé dans le cadre de la formation professionnelle initiale des EN du second degré. Il était plus précisément adressé aux EN considérés comme en "grande difficulté " dans leur travail suite aux visites d'aide et d'évaluation formative réalisées en milieu de formation (fin janvier) par respectivement un formateur et un membre du corps d'inspection disciplinaire. De manière complémentaire

4. Nous reprenons la terminologie institutionnelle. 
aux dispositifs de formation habituellement proposés aux EN, ce dispositif était constitué de deux volets. Un premier volet était effectué en institut de formation $^{5}$. Il était mené sous la forme de plusieurs séquences d'analyse de pratiques professionnelles dont l'objet principal était l'amélioration de la "Prise en main des élèves" par les EN. Un second volet était effectué en établissement scolaire à partir duquel l'étude a été réalisée. Ce volet a pris la forme d'une série de visites formatives impliquant le formateur de terrain (FT) ayant la responsabilité de l'EN au quotidien dans l'établissement et un des formateurs universitaires (FU) impliqués dans le premier volet de formation. Ces visites se structuraient typiquement autour de deux temps successifs: une leçon menée par l'EN avec sa classe sous l'observation conjointe du FT et du FU suivi d'un temps de formation de type entretien de conseil pédagogique (ECP) impliquant l'ensemble des acteurs de la triade. Il est à noter que le caractère aménagé de ces visites tenait dans la possibilité offerte aux formateurs, après s'être entendu avec l'EN, de le former à prendre en main et contrôler ses élèves en cours de leçon. Pour réaliser cette étude, nous nous sommes appuyés sur un total de trois visites formatives successives pour chacune des triades. Ces visites étaient espacées d'une à deux semaines.

\section{III.2. Participants}

L'étude de cas a été menée avec deux triades de formation volontaires. Ces triades étaient constituées d'une EN, d'une FT et d'une FU. Agées de 24 et 26 ans, les EN étaient des professeurs titulaires stagiaires de Français et de Mathématiques. Elles avaient pour la première fois la responsabilité de leurs propres classes pendant une année scolaire complète. Au moment de l'étude, leur expérience en enseignement était de 48 journées en établissement scolaire. Les deux FT étaient à la fois considérées comme des enseignantes expérimentées de Français et de Mathématiques (respectivement : 16 et 22 années d'expérience) mais aussi comme des FT expérimentées (cinq années d'expérience chacune dans cette fonction). Les deux FU étaient des enseignantes du second degré exerçant dans les départements disciplinaires de Français et de Mathématiques de l'IUFM. Elles avaient toutes les deux une expérience d'enseignante (respectivement 14 et 18 années) et de FT (respectivement 4 et 6 années).

\section{III.3. RECUEIL DES DONNÉES}

Pour réaliser cette étude, deux catégories de données ont été recueillies afin d'être retranscrites sous forme de verbatim et traitées. Pour chacune des visites, des données d'enregistrement ont d'abord été recueillies lors de la leçon puis de l'ECP. Une caméra vidéo et un micro HF ont été utilisés pour ce recueil. Des données dites d'autoconfrontation ont ensuite été recueillies au cours d'enregistrements audio-vidéo. À chaque

5. Dans l'académie considérée, la formation dite "universitaire " était déléguée par le Rectorat à l'Institut Universitaire de Formation des Maîtres (IUFM). 
visite de formation, chacun des acteurs de la triade a en effet réalisé un entretien d'autoconfrontation (EAC) relatif à l'ECP. Au total, 18 EAC, d'une durée de $45^{\prime}$ à une heure, ont été réalisés. Ces entretiens d'EAC ont été conduits de façon à pouvoir reconstituer a posteriori les règles suivies par les acteurs en cours d'ECP. Par un questionnement semi structuré, le chercheur incitait plus précisément l'acteur interviewé à l'instruire sur les significations à attribuer aux actions observées et aux jugements pouvant y être associés (par exemple : "Qu'est-ce que tu fais là ?"; "Qu'est-ce que tu penses de ton intervention à ce moment de l'entretien ?"). En lui demandant des précisions (par exemple: "Fe ne comprends pas pourquoi tu considères cette action comme intéressante, peux-tu reprendre?"), ou jouant sur la controverse en plaçant l'acteur interviewé face à des contradictions apparentes (par exemple: "Ce qui est étonnant, c'est que tu ne lui dis pas clairement comme tu me le dis à moi que ce n'est pas satisfaisant?"), le chercheur invitait ensuite l'acteur à étayer les jugements préalablement portés sur les actions signifiées. Enfin, il invitait l'acteur à préciser les résultats attendus quant aux actions observées (par exemple : "Et là tu t'attends à quoi?").

\section{III.4. TRAITEMENT DES DONNÉES}

Pour identifier et formaliser les règles apprises et/ou suivies par les différents acteurs au cours du dispositif de formation, nous avons adopté la procédure proposée par Chaliès, Bruno, Méard, \& Bertone (2010). Les données recueillies ont été traitées en quatre étapes successives.

(i) L'ensemble des six ECP et 18 EAC a été retranscrit en verbatim puis découpé en unités d'interaction. Ces unités ont été délimitées à partir de l'objet des significations attribuées par l'acteur autoconfronté aux événements visionnés. Une nouvelle unité d'interaction a été créée à chaque fois que l'objet de la signification attribuée par l'acteur changeait.

(ii) Pour chaque unité d'interaction, les éléments d'étayage de la signification attribuée par l'acteur autoconfronté sont ensuite identifiés. Par convention, ces éléments d'étayage correspondent à l'ensemble des circonstances évoquées par l'acteur pour expliquer au chercheur la façon de s'y prendre pour signifier de la même façon que lui, c'est-à-dire en suivant la même règle, les événements de la situation de formation visionnés.

(iii) Pour chaque unité d'interaction, la règle suivie par l'acteur pour comprendre son expérience et en juger est formalisée. Par convention, chaque règle est étiquetée à partir de (i) l'objet de la signification attribuée par l'acteur, (ii) de l'ensemble des circonstances évoquées par l'acteur pour étayer cette signification et (c) des résultats constatés et/ ou attendus. Dans sa forme, chaque règle est présentée ainsi : ["Objet de la signification" vaut dans les circonstances où "ensemble des circonstances évoquées pour étayer la signification" ce qui obtient comme résultat "ensemble des résultats constatés et lou attendus"]. Afin de minimiser les interprétations du chercheur, chacune des règles a été étiquetée au plus près du vocabulaire des acteurs. 
(iv) La validité des résultats obtenus a été testée. L'ensemble du corpus a été analysé de façon séparée par deux chercheurs. Tous les résultats obtenus ont ensuite été comparés et discutés par les deux chercheurs jusqu'à l'obtention d'un accord. Sur l'ensemble du corpus analysé, moins de $5 \%$ des éléments identifiés ont été source de désaccord entre les chercheurs et ont été rejetés.

\section{RÉSULTATS}

Les résultats développés ci-dessous ont été sélectionnés compte tenu de l'objet de l'étude. Ils contribuent à renseigner dans quelles circonstances l'activité de formation engagée par les FU et les FT a influencé la satisfaction professionnelle des EN. Dans le détail, le premier résultat permet d'illustrer le postulat selon lequel la satisfaction professionnelle des EN est tout d'abord alimentée par l'engagement des formateurs dans une activité de formation exhaustive (constituée d'un enseignement ostensif des règles aux formés, de l'accompagnement de leurs premiers suivis et d'explications ostensives) afin de leur faire apprendre de nouvelles règles. Dans ces circonstances de formation, la satisfaction apparaît en effet alors comme consubstantielle au constat par les EN des résultats attendus suite aux suivis de ces règles préalablement enseignées mais non encore apprises. Le second résultat permet d'illustrer le postulat selon lequel la satisfaction professionnelle des EN est aussi alimentée par l'engagement des formateurs dans une activité de formation les accompagnant dans l'interprétation de règles préalablement apprises et déjà suivies de façon insatisfaisante en situation de travail.

IV.1. Première Circonstance De Formation SOURCE DE SATISFACTION POUR LES EN : L'ACCOMPAGNEMENT PAR LES FORMATEURS DES PREMIERS SUIVIS DES RĖGLES PRÉALABLEMENT ENSEIGNÉES

Au cours de son EAC relatif à la leçon de la Visite Formative 3, l'EN de la Triade 1 a interpellé le chercheur sur le fait que, pour la première fois, elle a trouvé satisfaction ("je me dis : "enfin! Ouf, ça y est, c'est bon on y est arrivé ") à suivre les conseils des formateurs (Extrait 1).

\section{Extrait 1:}

$\mathrm{EN}$ : Là, vous voyez, ça marche. Ils (les élèves) écoutent.

Chercheur $(\mathrm{CH})$ : Qu'est ce qui marche là exactement?

$\mathrm{EN}$ : Ben en fait là je baisse ma voix en fait, et hop ça va vite, ils (les élèves) s'en rendent compte et hop ils s'arrêtent (de bavarder)...

$\mathrm{CH}:$ Et donc tu me dis...

$\mathrm{EN}$ : Ben là c'est quand même un soulagement. Dans ma tête là je me dis : "enfin! Ouf, ça y est, c'est bon on y est arrivé ".

$\mathrm{CH}: \mathrm{Ca}$ a l'air de te soulager...

$\mathrm{EN}$ : Ouais complètement... Ca a mis du temps mais là voilà on y est (...) 
$\mathrm{CH}$ : Mais tu cherchais à faire quoi... Enfin ce que tu n'arrivais pas à faire, c'est quoi finalement...

$\mathrm{EN}$ : Ben là c'est un truc qu'on (avec les formateurs) bosse en fait depuis le début (du dispositif d'aide). L'idée c'est pour contrôler, on joue le silence. C'est ça l'idée en fait.

$\mathrm{CH}$ : C'est-à-dire?

$\mathrm{EN}$ : Pour les (les élèves) faire écouter moi je montais (la voix) si vous voulez, de plus en plus, et eux (les élèves) en fait finalement ils montaient avec moi. Donc l'idée ça était de moins le faire au contraire. Mme A (la FU) m'a proposé de parler moins fort pour justement les (les élèves) obliger en fait à être plus attentifs... Ils écoutent plus en fait si je parle moins fort... Et là c'est, c'est ce qui se passe en fait. Ils écoutent plus... donc voilà je les contrôle mieux, c'est ce que je dois travailler.

$\mathrm{CH}:$ Et donc là si je comprends bien aujourd'hui c'est la première fois que ça marche en fait?

EN : Ouais. D'habitude c'est quand même pas aussi efficace...

Cet extrait permet de documenter deux principales idées. Tout d'abord, l'EN trouve satisfaction suite aux suivis d'une règle qui lui a été préalablement enseignée par la FU (Règle suivie : [ "Contrôler les élèves 》 vaut pour "jouer sur le silence " c'est-à-dire "parler moins fort aux élèves » ce qui obtient comme résultat qu'ils sont "plus attentifs" et "écoutent»]). Plus précisément, l'EN trouve satisfaction dans la situation d'enseignement considérée, car elle parvient en suivant la règle à constater les résultats que lui avait associé la FU lors de son enseignement ("ça marche. Ils écoutent", "voilà on y est ", "Et là ça y est (...) ils écoutent plus»). De façon complémentaire, cet extrait permet de constater qu'il a fallu du temps à l'EN pour parvenir à être satisfaite suite au suivis de la règle considérée ("ça a mis du temps", "D'habitude c'est quand même pas aussi efficace...»). Autrement dit, il a donc fallu l'engagement répété de l'EN dans des suivis de la règle préalablement enseignée par la FU pour parvenir à en constater les résultats attendus et finalement clôturer son apprentissage. À ce niveau de développement se pose la question relative à la nature de l'activité de formation engagée par la FU et la FT pour parvenir à cet apprentissage de la règle et par-là même à l'accroissement de la satisfaction de l'EN. L'extrait qui suit est emprunté à l'ECP réalisé lors de la Visite Formative 1 (trois semaines plus tôt). Il permet d'illustrer l'engagement de la FU dans une activité d'enseignement ostensif de la règle considérée (Extrait 2).

\section{Extrait 2:}

FU : T'as vraiment pas intérêt à essayer de toujours parler comme ça, toujours de plus en plus fort. Alors je sais pas si tu t'en rends compte mais vraiment tu finis par vraiment crier...(...). Donc si tu veux par rapport à ça, parce que là on est en plein sur ce qu'on essaye de travailler quand même. Là, ça permet de mieux contrôler la classe quand même... donc pour moi, après Isabelle (la FT) te dira certainement ce qu'elle en pense, ou elle l'a peut être déjà dit, mais pour moi c'est un peu l'inverse... Moi je t'inviterais à faire l'inverse en fait. Gagner le silence par le silence si tu veux. L'idée c'est de jouer sur la voix. Tu réduits... Et ils vont être obligés de s'arrêter (de bavarder), de tendre l'oreille. 
EN : Oui, mais c'est pas...

FU : Oui pas simple oui. C'est sûr mais... c'est quand même efficace. Tu baisses la voix jusqu'à ce qu'ils t'écoutent. Tu les obliges en quelque sorte à être attentifs...

La difficulté rencontrée par l'EN pour apprendre la règle considérée, et par-là même y trouver satisfaction, ne semble pas dépendante d'un engagement des formatrices dans une activité de formation inadaptée. À ce moment de l'entretien, la FU s'engage en effet dans une activité d'enseignement ostensif de la règle. Elle dresse un lien de signification entre, d'une part, l'énoncé de cette dernière ("Contrôler la classe ") et, d'autre part, des circonstances expérientielles décrites ("jouer sur la voix", "baisser la voix»). Elle précise par ailleurs les résultats qu'il est possible d'attendre suite au suivi de cette règle ("les élèves vont être obligés de s'arrêter (de bavarder) ", "d'être attentifs"). En ce sens, la FU rend possible un engagement de l'EN dans des premiers suivis de la règle avec des attentes, c'est-à-dire un engagement susceptible d'alimenter sa satisfaction professionnelle. À ce niveau de développement deux réserves peuvent toutefois être émises et venir expliquer la difficulté rencontrée par l'EN pour apprendre la règle considérée. La première est liée à la seule verbalisation par la FU des exemples constitutifs de la règle enseignée. Très éloignée par définition des circonstances effectives de travail de l'EN, cette description n'a peut être pas suffi à placer l'EN en capacité de s'engager dans des premiers suivis adéquats de la règle considérée. Directement liée à la précédente, la seconde réserve est liée à l'absence d'engagement de la FU dans des explications ostensives afin de sortir l'EN d'une éventuelle mésinterprétation et/ou incompréhension. L'extrait d'EAC de l'EN qui suit, relatif à ce moment de l'entretien, permet néanmoins d'écarter ces réserves. Il n'y a effectivement pas eu de mésinterprétation et/ou d'incompréhension de la part de l'EN lors de l'enseignement de la règle par la FU. Il permet également de mettre en évidence que, bien que l'activité d'enseignement de la règle ait été semble-t-il menée de façon adéquate ("là c'est plus clair. Fe sais ce qu'il faudrait faire") par la FU, elle n'alimente pas immédiatement ( $j$ 'attends de voir») la satisfaction de l'EN (Extrait 3).

\section{Extrait 3:}

$\mathrm{EN}$ : Là c'est plus clair. Je sais ce qui faudrait faire là.

$\mathrm{CH}$ : Je sais ce qu'il faut faire. C'est-à-dire ?

$\mathrm{EN}$ : Ben oui là elle (la FU) me dit d'arrêter d'essayer de parler toujours de plus en plus fort... Essayer de... Oui essayer de réduire en fait ma voix pour qu'ils (les élèves) écoutent plus en fait.

$\mathrm{CH}$ : Donc là tu as une solution en fait alors que tout à l'heure tu disais... (...) Tout à l'heure il n'y avait pas de solutions mais là y en a une non?

EN : Oui c'est vrai. Mais j'attends quand même de voir en classe. Là comme ça, ça paraît...

$\mathrm{CH}$ : Pas convaincue donc?

EN : Pas complètement. 
À ce niveau de développement, la question qui subsiste est celle relative aux circonstances qui ont finalement contribué à l'apprentissage de la règle considérée lors de la leçon de la Visite Formative 3. L'extrait d'EAC de la FT relatif à cette leçon permet d'y répondre (Extrait 4).

\section{Extrait 4:}

FT : Là c'est comme tout à l'heure. Je ne sais pas si tu as vu mais bon à chaque fois pour les consignes, à chaque fois qu'elle donne des consignes avec Dominique (la FU) on se fait plus présentes. Tout à l'heure d'ailleurs Dominique a même changé de place...(...)

$\mathrm{CH}$ : C'était donc prévu de l'aider comme ça.

FT : Oui là par rapport aux fois d'avant (les leçons antérieures) on (avec la FU) avait dans l'idée de lui simplifier ces moments où elle doit délivrer les consignes. Donc on se sépare, moi je me suis à un moment donné assise à côté des deux trois (élèves) agités... Mais on le lui (à l'EN) avait dit quand même. On lui avait demandé ça, si on pouvait un peu se montrer.

Cet extrait permet de constater que ce n'est pas seulement parce que l'EN s'est engagée dans des premiers suivis répétés de la règle que lui avait préalablement enseignée la FU qu'elle parvient tout à coup à clôturer son apprentissage et à s'en trouver satisfaite. L'engagement des formatrices dans une activité d'accompagnement de ces suivis a aussi, semblet-il, contribué à ce résultat. Contrairement à ce qu'elles faisaient jusqu'à présent, les formatrices ont en effet cherché lors de la leçon considérée à aménager la situation de travail de l'EN. Plus précisément, elles ont essayé de "simplifier" à l'EN les temps de passation des consignes aux élèves en s'efforçant d'être "plus présentes". En se montrant davantage ou se positionnant différemment dans la classe ("je me suis à un moment donné assise à côté des deux trois (élèves) agités "), les formatrices ont limité l'agitation des élèves. Elles ont par-là même permis à l'EN de s'engager dans des suivis de la règle enseignée mais aussi et surtout d'en constater les résultats attendus. Par leur engagement dans une activité de formation constituée d'un enseignement ostensif de la règle en situation de formation puis de l'accompagnement effectif de ses premiers suivis en situation de classe, les formatrices ont donc contribué à l'apprentissage de l'EN et alimenté sa satisfaction. La présence des formatrices dans la classe apporte ici la garantie d'un contrôle de l'action de l'EN en classe et constitue en soi un aménagement de la situation de travail qui, de ce fait, devient une situation de formation à part entière. C'est au cours de cette situation de formation/travail que l'EN apprend à réaliser une nouvelle action de métier avec l'aide d'autrui (Vygotski, 1985).

\section{IV.2. SECONDE CIRCONSTANCES DE FORMATION SOURCE DE SATISFACTION POUR LES EN : L'ACCOMPAGNEMENT PAR LES FORMATEURS DE L'INTERPRÉTATION DE RĖGLES APPRISES}

Au cours de l'EAC relatif à la leçon de la Visite Formative 2, l'EN de la Triade 2 a interpellé le chercheur sur le fait que les conseils des formatrices 
lui ont permis d'être un peu plus "efficace». À l'instant considéré de la leçon, elle est en effet d'autant plus satisfaite ("c'est plutôt bien là ", "ça marche quand même pas mal") de son activité aux côtés de ses élèves que ceux-ci ont pour la plupart réalisé le travail ("ils ont quand même tous bossé ») qu'elle leur avait donné (Extrait 5).

\section{Extrait 5 :}

EN : Là, ouf, c'est plutôt bien là... Y a quand même ce moment-là (dans la leçon). C'est ce que je vous disais tout à l'heure. Ils (les élèves) ont quand même tous bossé. Donc c'était dur... C'était pas. Pour moi avant là c'était dur. Mais là sur le temps où on essaye de faire la synthèse, là au tableau. Pour moi là, oui un espèce de ouf, ça marche quand même pas mal donc c'est...

$\mathrm{CH}$ : Oui c'est...

EN : C'est pas mal là... Là je dirais que c'est le meilleur moment. Je suis un peu plus efficace là...

Invitée par le chercheur à davantage expliciter les circonstances singulières de classe ayant conduit à cette satisfaction, l'EN précise en quelque sorte l'historique du travail de formation mené avec les formatrices sur la règle considérée (Extrait 6).

\section{Extrait 6:}

$\mathrm{CH}$ : Le temps de recherche vous l'aviez vu je ne me souviens pas de ça tu peux me préciser?

EN : Oui... Si vous voulez ce que je faisais moi c'était de faire comme on avait vu en fait avec Véronique (la FT). Je les (les élèves) mettais au travail et puis après je les interrogeais... Avec quand même l'idée d'alterner en fait. J'interrogeais un élève studieux disons et puis juste après un autre mais là plutôt, plus agité. Et comme ça bon on avançait quand même (dans le travail) et ils étaient plus attentifs... Pendant les exercices j'essayais de tenir la classe comme ça en fait...

$\mathrm{CH}$ : Donc ça c'était avant et là donc aujourd'hui tu as changé ?

$\mathrm{EN}$ : Ben là ... Comme moi, en fait, quand je faisais ça je trouvais que certains (élèves) ne travaillaient pas vraiment en fait. C'était pas complètement satisfaisant en fait... Donc après on en a parlé quand elles (les formatrices) l'ont vu et elles m'ont proposé de faire aussi attention au travail de recherche. D'être en fait surtout avec certains élèves avant... quand ils essayent de répondre. C'est ça que j'ai fait là (dans la leçon) mais c'était dur.

Lors du travail de formation engagé avec la FT avant la mise en place du dispositif d'aide, l'EN avait notamment appris la règle suivante : ["Tenir la classe pendant les exercices" vaut pour "interroger par alternance les élèves studieux et les élèves agités" ce qui obtient comme résultat de rendre les élèves "plus attentifs" et d' "avancer" dans le travail]. Lors du suivi de cette règle en classe, elle n'était toutefois pas "complètement satisfaite " du travail de l'ensemble des élèves (" certains (élèves) ne travaillaient pas vraiment en fait »). Elle a donc, semble-t-il, profité de l'entretien de la Visite Formative 
1 pour interpeller les formatrices sur cette difficulté. Ces dernières lui ont alors proposé une interprétation possible de la règle considérée en élargissant les éléments d'étayage la constituant ("faire aussi attention au travail de recherche ") afin qu'elle soit davantage satisfaite à l'occasion des suivis de cette dernière.

L'extrait qui suit est justement emprunté à l'ECP réalisé lors de la Visite Formative 1 (deux semaines plus tôt). Il est doublement illustratif. D'une part, il permet de documenter l'idée selon laquelle le manque de satisfaction de l'EN, suite aux suivis dans certaines circonstances de classe d'une règle préalablement apprise, est à l'origine du questionnement qu'elle adresse aux formatrices. D'autre part, il permet de mettre tout particulièrement en évidence l'engagement collectif de la FU et de la FT dans une activité d'interprétation de la règle considérée (Extrait 7).

\section{Extrait 7 :}

FT : Pour moi, le moment des exercices c'est nettement mieux. Ils (les élèves) sont beaucoup plus avec toi. Je trouve que c'est, c'est à noter quand même.

EN : Oui, en même temps. C'est pas, c'est pareil pour tous (les élèves). Malika par exemple. Et sa copine là, euh, Solenne. J'ai l'impression que ce sont en fait toujours les mêmes, quoi que je fasse eux...

FU : Ils te dérangent. Mais ce que vous (avec la FT) avez décidé à mon avis c'est quand même intéressant. Parce qu'en fait en changeant comme ça quand tu les questionnes ils sont obligés d'être un peu attentifs. Donc quelque part ça les tient.

EN : Oui c'est sûr, c'est mieux... Quand j'interroge c'est vrai ils sont un peu tenus mais... pour autant moi ce qui me gène c'est que certains ils n’ont pas vraiment travaillé le truc en fait. C'est moi souvent. Je réponds à leur place.

FU : C'est plus le travail en fait qui te... plus que le contrôle j'ai l'impression maintenant qui te...

EN : Oui. Moi je suis pas sûre qu'ils aient vraiment travaillé en fait. C'est quand même moi qui réponds en fait quand même là, pour certains.

Cet extrait permet de mettre en évidence que, insuffisamment satisfaite par les résultats obtenus suite au suivi d'une des règles apprises avec la FT, l'EN rentre en quelque sorte en controverse avec le point de vue porté par cette dernière sur l'activité des élèves lors du temps de la leçon consacré aux exercices. Alors que la FT considère que "c'est nettement mieux » en termes d'investissement des élèves à ses côtés, l'EN relève en effet que ce jugement ne peut être généralisé à toute la classe ("c'est pareil pour tous"). En précisant par ailleurs qu'elle est démunie de solutions ("quoi que je fasse ") pour répondre à la difficulté qu'elle rencontre ("je suis pas sûre qu'ils aient vraiment travaillé ", "C'est quand même moi qui répond"), elle sollicite indirectement l'aide des formatrices. C'est ce que confirme l'extrait de son EAC proposé ci-après. Lors de cet extrait, elle insiste sur le caractère encore non complètement satisfaisant des résultats obtenus ("ce qu'on (avec la FT) avait décidé ne marche pas tout le temps") suite aux suivis de la règle considérée. Elle s'engage aussi dans une explicitation de ce qu'elle a 
pu elle-même constater lors de la leçon avec comme intention première d'engager la discussion sur ce point ("je voudrais qu'on en discute ") et faire en sorte que des solutions soient trouvées pour suivre la règle de "façon peut être différente avec certains " élèves (Extrait 8).

\section{Extrait 8:}

EN : Là je reviens sur le fait que ce qu'on (avec la FT) avait décidé ne marche pas tout le temps en fait.

$\mathrm{CH}$ : Vous n'êtes pas d'accord on dirait ?

$\mathrm{EN}$ : Je sais pas. Là je voudrais qu'on en discute. OK alterner quand je questionne c'est pas mal mais pas pour tous (les élèves).

$\mathrm{CH}:$ Donc tu leur dis ça?

$\mathrm{EN}$ : Je dis ce que je ressens. Ce que je vois que peut être eux n'ont pas vu. $\mathrm{Tu} \ldots$

$\mathrm{CH}$ : C'est exprès là. Elle dit c'est bien et toi tu dis non pas tout à fait.

EN : Non moi là c'est pour mieux faire. Le faire de façon peut être différente avec certains (élèves).

Comme l'illustre l'extrait suivant, lors de son EAC relatif à cette séquence de formation, la FU suit la règle suivante : [ Enrichir la pratique de l'EN" vaut pour "confirmer que ce qui a été fait fonctionne" et "essayer de l'adapter pour chaque situation particulière " ce qui obtient comme résultat de " ne pas remettre tout en cause" et "ne pas déstabiliser l'EN"]. À l'instant considéré, son intention n'est donc pas de faire apprendre de nouvelles règles à l'EN mais plutôt de l'aider à "adapter " les suivis de règles préalablement apprises à la singularité des circonstances de classe rencontrées. Il est donc possible de considérer que la FU mène une activité de formation qui vise à soutenir le développement professionnel de l'EN en accompagnant son interprétation de règles préalablement apprises (Extrait 9).

\section{Extrait 9:}

FU : Ce qu'on fait là c'est pour pas. On veut pas tout remettre en cause en fait.

$\mathrm{CH}:$ Remettre en cause. C'est-à-dire ?

FU : Ce qu'elle fait n'est quand même pas complètement, complètement inefficace. Donc pour pas trop la déstabiliser on cherche plutôt en enrichir ce qu'elle fait. On va pas tout changer quoi.

$\mathrm{CH}$ : Et l'enrichir là pour toi c'est quoi là?

FU : Ben là on essaye en fait de... Bon déjà de confirmer que ce qui a été fait fonctionne si elle risque de. Et puis, après on essaye de trouver un moyen pour l'adapter.

Lors de son EAC relatif à cette même séquence de formation, la FT confirme qu'elle a, elle aussi, pour intention "d'améliorer ce que fait déjà » de façon satisfaisante l'EN. En plus des propos tenus par la FU, la FT précise l'interprétation qu'elle cherche à engager avec l'EN à propos de la règle qu'elle avait elle-même enseignée. En avançant une "solution qui complète " ce qui était fait jusqu'à lors, elle cherche donc à amener l'EN à suivre la 
même règle tout en l'interprétant comme suit: ["Tenir la classe pendant les exercices" vaut pour "préparer les réponses aux exercices avec les élèves agités" et "interroger par alternance les élèves studieux et les élèves agités " ce qui obtient comme résultat de rendre les élèves "plus attentifs" et d' "avancer " dans le travail]. L'extrait d'autoconfrontation de la FT qui suit documente ces idées (Extraits 10).

Extrait 10 :

$\mathrm{CH}$ : Là toi tu proposes une solution en fait ? C'est la première fois que tu interviens comme ça?

FT : Mais c'est une solution pour compléter en fait là. Je complète ce qui est dit par Béatrice (la FU).

$\mathrm{CH}:$ C'est mieux donc.

FT : Oui nettement. Mais là c'est plutôt essayer d'améliorer ce qui a été fait puisqu'elle (l'EN) nous dit que bon pour certains (élèves) c'est pas encore bien, bien concluant en fait... Par rapport au travail.

$\mathrm{CH}:$ Et donc toi là tu?

$\mathrm{FT}$ : Ben là pour lui répondre en fait. On... je lui dis t'as qu'à bosser directement avec eux (les élèves) quoi comme ça tu es sûre de...

$\mathrm{CH}:$ Bosser avec eux, c'est à dire...

FT : Oui elle peut peut-être avant de faire ce type d'échanges là, comme là aujourd'hui, elle peut travailler directement avec eux. Avant, pour préparer les réponses directement avec eux. Là ils travailleront si elle les fait travailler.

Finalement, par leur engagement dans une activité de formation accompagnant l'interprétation de règles préalablement apprises et déjà suivies de façon insatisfaisante en situation de travail par l'EN, les formatrices ont contribué à l'accroissement de la satisfaction et au développement professionnel de l'EN.

\section{DISCUSSION}

\section{V.1. ACCROÎTRE LA SATISFACTION PAR LA FORMATION : REPENSER LE PRINCIPE DE L'ALTERNANCE}

Les résultats présentés en amont permettent en premier lieu de souligner que la formation réflexive au travail n'est pas toujours une ressource suffisante pour les formés afin qu'ils agissent autrement en situation de travail. Plus en détails, ils permettent d'attester que l'impact des éléments dialogiques de réélaboration réflexive du travail en situation de formation sur le développement des "pouvoirs de l'action" (Clot, 2008) en situation de travail est, au moins pour partie, dépendant de la nature de l'aménagement de celle-ci. Le premier résultat montre la complexité de l'activité de formation engagée par les formatrices, qui ne se limite pas à un dialogue avant et/ou après la situation de travail. On constate que cette activité de formation déborde nettement la 
situation de formation à proprement parler pour se déployer aussi dans la situation de travail en classe. Ce déploiement de l'activité de formation en situation de travail apparaît d'ailleurs comme un "moment clé " au cours duquel un scénario particulier se joue. Aménagée par la présence des formatrices, la situation de travail " encourage " (Durand, 2008) alors l'EN à prendre des risques et à suivre les règles apprises en formation au sein même de la situation de classe. La présence des formatrices a notamment rendu l'action qui consistait à "baisser la voix » à la fois réalisable par l'EN et efficace en classe, y compris pour les élèves du fond de la salle qui, du coup, étaient à proximité des formatrices. C'est également dans ces circonstances que le résultat attendu ("les élèves vont être obligés d'arrêter de bavarder / d'être attentifs 》) a été atteint et constaté par l'EN, qui en a alors retiré une satisfaction. Autrement dit, la présente étude montre qu'une formation réflexive au travail peut contribuer à la réorganisation de l'activité décisionnelle située des formés ainsi qu'à l'augmentation de leur efficacité et de leur satisfaction professionnelle en situation de travail. C'est notamment le cas si le travail, aménagé en espace fictif intermédiaire de formation "par " et "au " travail (Durand \& Filletaz, 2009), est appréhendé dans la cadre d'un principe de continuité avec la formation. Il est alors inapproprié de parler "d'alternance " dans ces circonstances de travail - formation.

Ce constat est d'ailleurs déjà pour partie documenté par la littérature. On le retrouve dans la formation initiale des enseignants, où la présence physique en classe des FT et FU alimente l'activité décisionnelle des EN, jusqu'à les amener à infléchir leur plan de leçon, ce qui est inhabituel chez eux (Bertone, Méard, Ria, Euzet, et Durand, 2003). Des travaux récents ont également permis de montrer que cette dynamique de formation "par " et "au» travail est à la fois salutaire pour les EN et pour leurs tuteurs (Cartaut \& Bertone, 2009). En effet, ces derniers sont amenés à repenser et renormaliser leur propre travail en vue d'en faire un instrument de formation en direction des EN, soit pour tenir conseil, soit pour se "donner à voir " en classe au travers de pratiques inhabituelles pour eux mais qu'ils considèrent pourtant fécondes pour les formés dont ils ont la responsabilité. Le constat pointé en amont a pu aussi être établi dans d'autres secteurs du travail humain. C'est le cas par exemple des ouvriers du bâtiment (Duboscq \& Clot, 2010) qui parviennent à prendre de nouvelles décisions, dans la situation spécifique de "travail à l'échelle " pour l'élingage de panneaux en béton, suite à une controverse à propos des différentes façons de s'y prendre et des préoccupations correspondantes. On constate aussi la possibilité d'un développement professionnel des experts sous l'effet de la controverse dialogique accompagnée d'une gestuelle qui permet de mimer les opérations réalisées et/ou à réaliser dans des circonstances de visionnage du travail réel de chacun des opérateurs.

Pour conclure sur ce point, il est à noter que l'interprétation psychologique de ce développement salutaire du pouvoir d'agir proposée par la clinique de l'activité, est fidèle au concept vygotskien, de "migration fonctionnelle de l'expérience" (Vygotski, 2003) et s'appuie sur les propriétés de "liaison " entre expériences, propres à la conscience. Cette liaison entre expériences permettrait à une expérience passée de changer de statut : de situation vécue, au passé, l'expérience deviendrait, au moyen du dialogue, 
l'instrument pour vivre d'autres expériences présentes. L'un des mérites des résultats de cette étude est précisément de réhabiliter cette hypothèse de la migration fonctionnelle de l'expérience dialogique en formation initiale des enseignants. En effet, cette hypothèse ne trouvait pas de confirmations dans les données empiriques recueillies depuis une dizaine d'années. $\mathrm{Au}$ contraire, une étanchéité relative était régulièrement constatée entre, le développement à grands pas des capacités réflexives et d'analyse chez les EN en situation de formation, d'une part, et la stagnation de leurs possibilités de réalisation d'actions (conformes aux avancées de la formation) en situation de travail en classe, d'autre part (voir pour une synthèse Chaliès, Cartaut, Escalié, \& Durand, 2009). En matière de satisfaction au travail ce constat permet de fonder l'idée que la présence des formateurs en situation de travail en classe a engendré non seulement un contrôle des pratiques de l'EN, en facilitant l'émergence d'actions conformes aux attentes (Williams, 2002), mais a également permis l'émergence de critères de satisfaction inconnus auparavant par cette dernière. Ces considérations sont d'ailleurs conformes à l'idée selon laquelle les "tenants et aboutissants" des actions de métier sont " invisibles " pour les novices alors même que pour les experts ils renvoient à "des problèmes "réglés" à tous les sens du terme" (Clot, 2008, p. 263). Dans ces conditions, nous dit l'auteur, "celui qui entre dans une situation de travail sans la connaître n'a pas d'autre choix que de se tenir d'abord à la prescription qui est initialement sa seule ressource pour parvenir à faire ce qui est à faire. (...) C'est même au départ la source principale de l'action à laquelle le novice va emprunter ses raisons d'agir" (Ibid. p. 262).

\section{V.2. ACCROÎTRE LA SATISFACTION PAR LA FORMATION : CONCOURIR PAR L'ENSEIGNEMENT DE RÈGLES A L'ÉMERGENCE DE CRITÈRES DE SATISFACTION}

L'interprétation selon laquelle la satisfaction de l'EN a été le résultat d'une efficience accrue et de l'aboutissement de "pré-occupations anciennes " dans et par la réalisation "d'occupations nouvelles" (Saujat, 2010) paraît à première vue tout à fait plausible. Dans notre cadre conceptuel, il n'est toutefois pas possible de distinguer entre préoccupations et occupations, de sorte qu'une action pourrait changer et répondre ainsi à des préoccupations (stables ou inchangées) auxquelles l'action précédente n'avait pas pu répondre. Le caractère consubstantiel des préoccupations et des intentions avec l'action qui les incarne à chaque instant nous incite plutôt, au sein de cette étude, à considérer dans le même temps l'amélioration de l'efficacité de l'action (les occupations) et la satisfaction de préoccupations nouvelles. S'il est vrai que la satisfaction du travail bien fait dépend en large partie du constat que les résultats attendus sont obtenus, il est tout aussi vrai que le fait de porter un jugement sur l'atteinte des résultats suppose la présence de critères de jugement appris. Sur ce point, on suivra Ogien (2007) lorsqu'il affirme que toute connaissance pratique émerge d'une familiarisation avec des critères de jugement propres à une communauté de pratiques.

Ce en quoi nos résultats diffèrent de ceux généralement mobilisés en clinique de l'activité pour l'analyse du travail humain, c'est précisément 
qu'ils portent sur des travailleurs qui sont des novices et non des experts déjà familiarisés avec des critères de jugement du travail bien fait, capables d'apprécier le degré de satisfaction de leur activité, voire d'en discuter au cours de "débats d'école " (Clot, 2010). Pour pouvoir recycler des préoccupations vitales, les novices doivent d'abord et avant tout pénétrer dans un monde de préoccupations qui sont à la fois nouvelles et extrinsèques pour eux, tout en apprenant à suivre simultanément les règles du métier lui-même. Pour juger de l'efficacité de leurs actions et éventuellement s'en trouver satisfaits, ils ne peuvent donc initialement s'en tenir qu'aux règles du métier et aux critères de jugement appris en même temps que celles-ci. C'est en ce sens que la satisfaction des novices quant à leur efficacité, est en même temps et de façon consubstantielle une satisfaction de critères publics appris et l'atteinte de résultats attendus selon ces mêmes critères.

Le premier résultat documenté dans cette étude montre précisément l'émergence, sous contrôle des formatrices, d'une action à la fois nouvelle, conforme aux attentes et permettant d'atteindre des résultats qui ne sont qu'en apparence les mêmes que ceux que l'EN visait déjà auparavant. Bien que subtile, la différence apparaît grâce au grain d'analyse adopté dans cette étude. Il révèle en effet la complexité de la nouvelle préoccupation qui a émergé de cet apprentissage de la règle ["Contrôler les élèves" vaut pour "jouer sur le silence " c'est-à-dire "parler moins fort aux élèves » ce qui obtient comme résultat qu'ils sont "plus attentifs" et qu'ils "écoutent»]. C'est justement le fait que la nouvelle préoccupation (les élèves doivent être attentifs) n'est toujours pas atteinte, qui rend la satisfaction de l'EN seulement partielle dans les circonstances de classe étudiées. En d'autres termes, émergeant de façon consubstantielle avec l'action qui consiste à "baisser la voix ", la préoccupation liée à la captation de l'attention des élèves se révèle non satisfaite et met l'EN en situation de souhaiter autre chose que le résultat obtenu (les élèves arrêtent de bavarder). C'est précisément qu'être attentif ne suppose pas seulement d'arrêter de bavarder, mais aussi de "travailler", " écouter", "apprendre ", etc., autant de préoccupations nouvelles indexées à l'émergence de l'occupation nouvelle. Dans notre vocabulaire, nous dirons que le suivi d'une règle nouvelle, qu'elle ait été explicitement formulée ou non, modifie le jugement et permet de dire ce qui est correct, satisfaisant, conforme aux attentes instituées (Wittgenstein, 2004). de manière complémentaire, le deuxième résultat développé documente pour l'essentiel l'hypothèse que la satisfaction professionnelle en formation initiale a ceci de spécifique, au regard des interactions entre experts : l'émergence même des critères de satisfaction est consubstantielle de l'apprentissage des règles de métier et des capacités normatives de jugement de son action.

\section{V.3. ACCroÎtre La SATISFACTION PAR LA Formation : ACCENTUeR LA QUALITÉ DU TRAVAIL PLUTÔT QUE SON RENDEMENT}

Dans le prolongement de travaux récents portant sur les rapports entre santé et travail (par exemple : Miossec \& Clot, 2011), les résultats présentés en amont en appellent aussi à discuter le caractère hégémonique de 
l'approche psychosociale de la satisfaction comme correspondant d'une perception d'autoefficacité. Certes la perception d'efficacité personnelle peut être source de satisfaction, mais il est également avéré qu'elle peut être tout autant vécue comme un échec psychologique pour le sujet (Clot, 2010). Corrélée à une dégradation de la qualité du travail et de l'utilité sociale du produit de ce travail, l'efficacité n'est alors que "rendement" vide de sens (Sarnin, Caroly, \& Douiller, 2011). Il en va de même pour toutes ces situations où, sous prétexte de rationalisation du travail, les référentiels de "bonnes pratiques " remplacent des procédures qui ont fait leurs preuves et dépossèdent les opérateurs de leurs propres pratiques et concepts pour faire et penser le métier.

Dans ces circonstances, selon Clot (2010), "c'est l'instrument collectif (...) qui est maltraité; les gestes traditionnels qui sont disqualifiés sans l'inventaire patient auquel les agents pourraient pourtant prêter la main. Car c'est aussi un plaisir de transformer son expérience en moyen d'en vivre une autre " (p. 54), de même que "bien faire quelque chose est source de joie" (p. 40). Le plaisir et la satisfaction du travail bien fait résistent aux seules considérations quantitatives d'efficience au travail et de conformité aux bonnes pratiques. Selon l'auteur, c'est bien à ce niveau que les travailleurs expérimentés souffrent du sale boulot qu'on leur demande de faire, tant au niveau des pratiques standardisées qu'ils subissent, que des résultats que ces pratiques produisent en termes de "qualité empêchée ». Et c'est bien à ce niveau que le sentiment d'efficacité ne permet plus de comprendre les ressorts de la souffrance et de la satisfaction au travail.Vus sous cet angle, les résultats de cette étude montrent alors la très grande fragilité des novices au regard d'une conception gestionnaire du travail fondée sur des référentiels de compétences. Ils répondent en partie aux réserves régulièrement émises sur la pertinence des formations qui ne pénètrent pas la réalité du métier et ne préparent pas suffisamment les novices au "sale boulot" qu'ils doivent pourtant apprendre à faire (Cattonar, 2006 ; Maroy \& Cattonar 2002). En apportant une aide inscrite dans les circonstances mêmes de l'exercice du métier au quotidien, les formateurs peuvent accompagner de façon efficace les EN dans la réalisation d'actions de métier pour lesquelles ils ne sont pas formés en centre universitaire.

Ce qui attend le métier enseignant, avec des formations de plus en plus normatives et des évaluations indexées à un référentiel de compétences qui n'a plus rien d'un instrument collectif pour faire le métier, laisse présager une perte de sens et de plaisir au travail. Les prémisses d'une "qualité empêchée " sont toutes présentes en ce qui concerne la formation des enseignants, prémisses accentuées pas la réforme dite de "la mastérisation " de l'enseignement qui a été pensée dans le déni de toutes les avancées patiemment capitalisées par des générations de FT et FU. En montrant que la qualité du travail n'est pas seulement une question de rendement, mais aussi de satisfaction, de joie et de réalisation de soi, cet article milite au contraire pour une formation initiale des enseignants pensée sur la base des règles du métier davantage que sur les référentiels de compétences. Le pari n'est pas celui du "plaisir contre l'efficience ", mais celui du développement d'une " conscience professionnelle » comme « facteur de santé autant que d'efficacité, quand elle 
est entretenue " (Clot, 2010, p. 44). Et si le rôle de l'organisation du travail est bien d'entretenir cette conscience professionnelle, c'est en amont celui de la formation initiale que de la faire émerger chez les novices.

\section{RÉFÉRENCES}

Berducci, D. (2004). Vygotsky through Wittgenstein: A New Perspective on Vygotsky's developmental continuum. Theory Psychology, 14, 329-353.

Bertone, S., Chaliès, S., \& Clot, Y. (2009). Contribution d'une théorie de l'action à la conceptualisation et à l'évaluation des pratiques réflexives dans des dispositifs de formation initiale des enseignants. Le Travail Humain, 72, 104-125.

Bertone, S., Méard, J.A., Ria, L., Euzet, J.P., \& Durand, M. (2003). Intrapsychic conflict experienced by a preservice teacher during classroom interactions : A case study in physical education. Teaching and Teacher Education, 19, 113-125.

Bertrand, F., Peters, S., Pérée, F., \& Hansez, I. (2010). Facteurs d'insatisfaction incitant au départ et intention de quitter le travail : analyse comparative des groupes d'âges. Le Travail Humain, 73, 213-237.

Brunetti, G.J. (2001). Why do they teach? A study of job satisfaction among longterm high school teachers. Teacher Education Quarterly, 28, 49-74.

Caprara, G.V., Barbaranelli, C., Steca, P., \& Malone, P.S. (2006). Teachers' selfefficacy beliefs as determinants of job satisfaction and students' academic achievement. Fournal of School Psychology, 44, 473-490.

Cartaut, S., \& Bertone, S. (2009). Co-analysis of work in the triadic supervision of preservice teachers based on neo-vygotskian activity theory : case study from a French University Intitute of Teacher Training. Teaching and Teacher Education, 25, 1086-1094.

Cash, M. (2009). Normativity is the mother of intention:Wittgenstein, normative practices and neurological représentations. New Ideas in Psychology, 27, 133147.

Cattonar, B. (2006). Convergence et diversité professionnelle des enseignantes et des enseignants du secondaire en Communauté française de Belgique : tensions entre le vrai travail et le sale boulot. Éducation et Francophonie, 34(1), 193-212.

Chaliès, S., Bruno, F., Méard, J., \& Bertone, S. (2010). Training preservice teachers rapidly: the need to articulate the training given by university supervisors and cooperating teachers. Teaching and Teacher Education, 26, 764-774.

Chaliès, S., Cartaud, S., Escalié, G., \& Durand, M. (2009). Note de synthèse - D'une utilité discutée à une utilité éprouvée du tutorat en formation initiale des enseignants : la nécessité d'une formation des tuteurs. Recherche et Formation, 61, 85-129.

Chauviré, C. (Ed.). (2010). Wittgenstein en héritage. Philosophie de l'esprit, épistémologie, pragmatisme. Paris : Kimé.

Ciftci, A.M., Ozkan Ozgun, O., \& Erden S. (2011). Self-efficacy and satisfaction of pre-service early childhood education teachers as a function of perceived needs and experiences. Procedia Social and Behavioral Sciences, 15, 539-544.

Clot, Y. (2008). Travail et pouvoir d'agir. Paris : PUF.

Clot, Y. (2010). Le Travail à cour. Pour en finir avec les risques psychosociaux. Paris : La Découverte.

Clot, Y., \& Soubiran, M. (1998). "Prendre la classe » : une question de style ? Société française, $12 / 13,78-88$.

Davis, A. (2009). Examples as method ? My attempts to understand assessment and fairness in the spirit of the later Wittgenstein. Fournal of Philosophy of Education, 43, 371-389. 
Day, C., Sammons, P., Stobard, G., Kington, A., \& Gu, Q. (Eds.). (2007). Teachers matter: Connecting work, lives and effectiveness. Berkshire: Open University Press.

Demirtas, Z. (2010). Teachers' job satisfaction levels. Procedia Social and Behavioral Sciences, 9, 1069-1073.

Descombes, V. (1996). Les Institutions du sens. Paris: Éditions de Minuit.

Descombes, V. (2004). Le Complément de sujet: enquête sur le fait d'agir de soi-même. Paris: Gallimard.

Duboscq, J., \& Clot, Y. (2010). L'autoconfrontation croisée comme instrument d'action au travers du dialogue : objets, adresses et gestes renouvelés. Revue d'Anthropologie des Connaissances, 4, 255-286.

Duffy, R.D., \& Lent, R.W. (2009). Test of a social cognitive model of work satisfaction in teachers. Fournal of Vocational Behavior, 75, 212-223

Durand, M. (2008). Un programme de recherche technologique en formation des adultes. Une approche enactive de l'activité humaine et l'accompagnement de son apprentissage/développement. Éducation et Didactique, 2, 1-25.

Durand, M., \& Filliettaz, L. (Eds.). (2009). Travail et formation des adultes. Paris: PUF.

Fabra, M.E., \& Camison, C. (2009). Direct and indirect effects of education on job satisfaction: A structural equation model for the Spanish case. Economics of Education Review, 28, 600-610

Faragher E.B, Cass M., \& Cooper C.L. (2005). The relationship between job satisfaction and health: a meta-analysis. Occup Environ Med, 62, 105-112.

Gauvry, C. (2010). Les limites de la lecture externaliste du meinen chezWittgenstein: Une intentionnalité grammaticale. Bulletin d'Analyse Phénoménologique, 6(8), 129-143.

Hansez, I., Bertrand, F., De Keyser, V., \& Pérée, F. (2005). Fin de carrière des enseignants : vers une explication du stress et des retraites prématurées. Le Travail humain, 68, 193-223.

Hastings, R.P., \& Bham, M.S. (2003). The relationship between student behavior patterns and teacher burnout. School Psychology International, 24, $115-127$.

Huang, S.Y., \& Waxman, H.C. (2009) The association of school environment to student teachers' satisfaction and teaching commitment. Teaching and Teacher Education, 25, 235-243

Kyriacou, C. (2001). Teacher stress: Directions for future research. Educational Review, 53, 27-35.

Laugier, S., \& Chauviré, C. (Eds.). (2006). Lire les recherches philosophiques de Wittgenstein, Paris : Vrin.

Le Blanc, G. (Éd.). (2004). Les Maladies de l’homme normal. Bègles : Éditions du Passant.

Liu, X.S., \& Ramsey, J. (2008) Teachers' job satisfaction: Analyses of the Teacher Follow-up Survey in the United States for 2000-2001. Teaching and Teacher Education, 24, 1173-1184

Lave, J., Wenger, E. (Eds.). (1991). Situated learning: Legitimate peripheral participation. Cambridge: Cambridge University Press.

Ma, X., \& MacMillan, R. (1999). Influences of workplace conditions on teachers' job satisfaction. The fournal of Educational Research, 93, 39-47.

Maroy, C., \& Cattonar, B. (2002). Professionnalisation ou déprofessionnalisation des enseignants? Cahier de Recherche du GIRSEF, 18, 1-27.

Miossec, Y., \& Clot, Y. (2011). Le métier comme instrument de protection contre les risques psychosociaux au travail: le cas d'ingénieurs managers de proximité. Le Travail Humain, 74(4), 341-363.

Moè A., Pazzaglia, F., \& Ronconi, L., (2010). When being able is not enough. The combined value of positive affect and self-efficacy for job satisfaction in teaching. Teaching and Teacher Education, 26, 1145-1153. 
Narboux, J.P. (2003). Incommensurabilité et exemplarité : aliénation logique et problème des universaux. Archives de Philosophie, 66, 437-447.

Nelson, K. (2008). Wittgenstein and contemporary theories of word Learning. New Ideas in Psychology, 4, 1-13.

Ogien, A. (Éd.). (2007). Les Formes sociales de la pensée. La sociologie aprèsWittgenstein. Paris : Armand Colin.

Papanastasiou, E.C., \& Zembylas, M. (2005). Job satisfaction variance among public and private kindergarten school teachers in Cyprus. International fournal of Educational Research, 43, 147-167

Pearson, L.C., \& Moomaw, W. (2006). Continuing validation of the Teacher Autonomy Scale. Fournal of Educational Research, 100, 44-51.

Rogoff, B., Matusov, E., \& White, C. (1996). Models of teaching and learning: participation in a community of learners. In D.R. Olson \& N. Torrance (Eds.), The handbook of education and human development: New models of learning, teaching and schooling (pp. 388-414). Oxford: Basil Blackwell Publishers.

Rots, I., Aelterman, A., Devos, G., \& Vlerick, P. (2010). Teacher education and the choice to enter the teaching profession: A prospective study. Teaching and Teacher Education, 26, 1619-1629.

Roussel, P. (Ed.). (2001). La Motivation au travail: concepts et théories. Paris: Management et société.

Saujat, F. (2010). Travail, formation et développement des professionnels de l'éducation. Note de synthèse pour l'HDR. Université d'Aix-Marseille I.

Sarnin, P., Caroly, S., \& Douillet, P. (2011). Contre les "risques " psychosociaux, quel débat sur l'activité ? Le Travail humain, 74, 309-320.

Shen, J., Leslie, J.M., Spybrook, J.K., \& Ma, X. (2011). Are principal background and school process related to teacher job satisfaction? A multilevel study using schools and staffing survey 2003-04. American Educational Research fournal, 48, 1-31.

Skaalvik, E.M., \& Skaalvik, S. (2011). Teacher job satisfaction and motivation to leave the teaching profession: Relations with school context, feeling of belonging, and emotional exhaustion. Teaching and Teacher Education, 27, 1029-1038.

Smylie, M.A. (1994). Redesigning Teachers'Work: Connections to the Classroom. Review of Research in Education, 20, 129-177

Vygotski, L.S. (1985). Pensée et Langage. Paris: Terrains/Éditions Sociales.

Vygotski, L.S. (Ed.). (2003). Conscience, inconscient, émotions. Paris : La Dispute.

Weiss, H.M. (2002). Deconstructing job satisfaction: separating evaluations, beliefs and affective experiences. Human Resource Management Review, 22, 173-194.

Williams, M. (2002). Tout est-il interprétation ? In C. Chauviré \& A. Ogien (Eds.), La régularité (pp.207-233). Paris : EHESS.

Winch, P. (Ed.). (2009). L'Idée d'une science sociale et sa relation à la philosophie. Paris : Gallimard.

Wittgenstein, L. (Éd.). (2004). Recherches philosophiques. Paris : Gallimard.

Yvon, F. \& Clot, Y. (2003). Apprentissage et développement dans l'analyse du travail enseignant. Pratiques Psychologiques, 1, 19-35.

\section{RÉSUMÉ EN FRANÇAIS}

Bien qu'il soit admis qu'elle a une influence sur la santé des enseignants, seuls quelques travaux ont montré un lien de causalité entre la satisfaction professionnelle des enseignants et la possibilité qui leur est offerte de se former. $\dot{A}$ partir d'un cadre conceptuel inspiré des théories générales de l'apprentissage (Berducci, 2004; Nelson, 2008) et de l'action collective (Wittgenstein, 2004), et de données d'autoconfrontation à propos de l'expérience vécue 
de classe et de formation, cette étude montre que l'activité de formation joue un rôle crucial sur la construction des règles de métier et des critères d'appréciation par les novices de la qualité de leur travail. Elle documente également l'idée selon laquelle c'est au cœur de la situation de travail ellemême, aménagée en situation de formation, que l'émergence de ces règles et critères est facilitée et que les formés éprouvent du plaisir à faire leur travail selon les règles de métier. Outre le fait de discuter l'efficacité du principe de l'alternance, cette étude propose finalement trois orientations pour parvenir à accroître par la formation la satisfaction professionnelle des enseignants novices.

Mots-clés : Formation; principe de l'alternance ; satisfaction professionnelle ; travail ; règles de métier ; enseignants novices.

Manuscrit reçu : juillet 2012

Accepté par N. Michinov après révision : mars 2013 